\title{
Tits Alternative for Maximal Subgroups of Skew Linear Groups
}

\author{
D. Kiani, M. Mahdavi-Hezavehi \\ dkiani@aut.ac.ir, mahdavih@sharif.edu
}

\begin{abstract}
Let $D$ be a noncommutative finite dimensional $F$-central division algebra, and let $N$ be a normal subgroup of $G L_{n}(D)$ with $n \geq 1$. Given a maximal subgroup $M$ of $N$, it is proved that either $M$ contains a noncyclic free subgroup or there exist an abelian subgroup $A$ and a finite family $\left\{K_{i}\right\}_{1}^{r}$ of fields properly containing $F$ with $K_{i}^{*} \subset M$ for all $1 \leq i \leq r$ such that $M / A$ is finite if Char $F=0$ and $M / A$ is locally finite if $\operatorname{Char} F=p>0$, where $A \subseteq K_{1}^{*} \times$ $\cdots \times K_{r}^{*}$.
\end{abstract}

\section{Introduction}

Let $D$ be a finite dimensional $F$-central division algebra. Denote by $M_{n}(D)$ the $n \times n$ matrix ring over $D$ and $S L_{n}(D)$ the commutator subgroup of the multiplicative group $G L_{n}(D)=M_{n}(D)^{*}$. Let $N$ be a normal subgroup of $G L_{n}(D)$ with $n \geq 1$. Given a subgroup $M$ of $N$, we shall say that $M$ is maximal in $N$ if for any subgroup $H$ of $N$ with $M \subset H$, one concludes that $H=N$. Now, let $D$ be a noncommutative division ring not necessarily of finite dimension over its centre $F$. The problem of whether $G L_{n}(D)$ contains a noncyclic free subgroup seems to be posed first by Lichtman in [11]. Stronger versions of this problem which essentially deal with the existence of noncyclic free subgroups in normal or subnormal subgroups of $G L_{n}(D)$ have been investigated in [6] and [7]. It is known so far that these problems have positive answers as long as we work in a division algebra of finite dimension over its 
centre. Further investigations for the infinite dimensional case are also dealt with in those articles. Given a maximal subgroup $M$ of $G L_{n}(D)$, it is proved in [12] that either $M$ contains a noncyclic free subgroup or there exists a finite family $\left\{K_{i}\right\}_{1}^{r}$ of fields with $F^{*} \subset K_{i}^{*} \subset M$ for all $1 \leq i \leq r$ such that $M / A$ is finite if $C h a r F=0$ and $M / A$ is locally finite if $\operatorname{Char} F=p>0$, where $A=K_{1}^{*} \times \cdots \times K_{r}^{*}$. Now, let $N$ be a normal subgroup of $G L_{n}(D)$ with $n \geq 1$, and $M$ be a maximal subgroup of $N$. The aim of this note is to investigate the structure of maximal subgroups of $N$ with respect to non-cyclic free subgroups sitting in $M$. To be more precise, let $D$ be a noncommutative division algebra of finite dimension over its centre $F$. Given a maximal subgroup $M$ of $N$, it is proved that either $M$ contains a noncyclic free subgroup or there exist an abelian subgroup $A$ and a finite family $\left\{K_{i}\right\}_{1}^{r}$ of fields with $F^{*} \subset K_{i}^{*}$ for all $1 \leq i \leq r$ such that $M / A$ is finite if $C h a r F=0$ and $M / A$ is locally finite if $C h a r F=p>0$, where $A \subseteq K_{1}^{*} \times \cdots \times K_{r}^{*}$. This, in particular, generalizes the main result of [12] concerning maximal subgroups of $G L_{n}(D)$.

\section{Notations and conventions}

Let $D$ be an infinite $F$-central division ring and assume that $N$ is a non-central normal subgroup of $G L_{n}(D)$ with a maximal subgroup $M$. Given a subgroup $G$ of $G L_{n}(D)$, we denote by $F[G]$ the $F$-algebra generated by elements of $G$ over $F$. We also denote by $D^{n}$ the space of row $n$-vectors over $D$. Then $D^{n}$ is a $D-G$ bimodule in the obvious manner. $G$ is said to be an irreducible (reducible) subgroup of $G L_{n}(D)$ whenever $D^{n}$ is irreducible (reducible) as $D-G$ bimodule. Considering the elements of $D^{n}$ as column vectors, we may regard $D^{n}$ as a $G-D$ bimodule. It is easily shown that $D^{n}$ is irreducible (reducible) as a $G-D$ bimodule precisely when it has the property as $D-G$ bimodule. We shall say that $G$ is absolutely irreducible if $M_{n}(D)=F[G]$. For any group $G$ we denote its centre by $Z(G)$. Given a subgroup $H$ of $G, N_{G}(H)$ means the normalizer of $H$ in $G,[G: H]$ denotes the index of $H$ in $G$, and $\langle H, K\rangle$ the group generated by $H$ and $K$, where $K$ is a subgroup of $G$. We shall say that $H$ is soluble-by-finite if there is a soluble normal subgroup $K$ of $H$ such that $H / K$ is finite. Let $S$ be a subset of $M_{n}(D)$, then the centralizer of $S$ in $M_{n}(D)$ is denoted by $C_{M_{n}(D)}(S)$. We shall identify the centre $F I$ of $M_{n}(D)$ 
with $F$. For each $d \in D^{*}$, denote by $A_{d}$ the matrix obtained from the unit matrix by replacing the $(1,1)$-th and $(n, n)$-th entries with $d$ and $d^{-1}$, respectively. Some notations and conventions for linear groups and skew linear groups from [18], [21] and [16] are frequently used throughout.

\section{Maximal subgroups of normal subgroups in $G l_{n}(D)$}

Given a division ring $D$ with center $F$, let $N$ be a normal subgroup of $G L_{n}(D)$. This section essentially deals with the structure of maximal subgroups $M$ of $N$ and how they sit in $N$ with respect to noncyclic free subgroups. To prove our main result, we shall need some commutativity theorems that enable us to understand better the structure of $M$. To be more precise, given an $F$-central division ring $D$, let $N$ be a non-central normal subgroup of $G L_{n}(D)$ with $n \geq 1$. Assume that $M$ is a maximal subgroup of $N$. It is shown that either $M$ is irreducible or there exists $P \in G L_{n}(D)$ such that $P^{-1} A_{d} P \in M$ for any $d \in D^{*}$. Using this result, it is also proved that if $D$ is infinite, then there exists no non-abelian maximal subgroup $M$ of $N$ such that $\left|M / M \cap F^{*}\right|<\infty$. We then show that $M$ is nilpotent if and only if $M$ is contained in the multiplicative group of a subfield of $M_{n}(D)$. Finally, using above results as well as various other results from algebraic group and skew linear group theory, it is proved that either $M$ contains a noncyclic free subgroup or there exist an abelian subgroup $A$ and a finite family $\left\{K_{i}\right\}_{1}^{r}$ of fields with $F^{*} \subset K_{i}^{*}$ for all $1 \leq i \leq r$ such that $M / A$ is finite if $C h a r F=0$ and $M / A$ is locally finite if $C h a r F=p>0$, where $A \subseteq K_{1}^{*} \times \cdots \times K_{r}^{*}$. We begin our study with the following lemmas:

Lemma 1. Let $D, N$, and $M$ be as above. Then either $M$ is irreducible or there exists $P \in G L_{n}(D)$ such that $P^{-1} A_{d} P \in M$ for any $d \in D^{*}$.

Proof. If $n=1$, then $M$ is clearly irreducible. Thus, we may assume $n \geq 2$. If $M$ is reducible, then there exists an invertible matrix $P$ and a natural number 
$0<m<n$ such that

$P M P^{-1} \subseteq H=\left\{\left[\begin{array}{cc}A & B \\ 0 & C\end{array}\right] \mid A \in G L_{m}(D), C \in G L_{n-m}(D), B \in G L_{m \times(n-m)}(D)\right\}$.

By maximality of $M$ in $N$, two cases may occur. If $H \cap N=P M P^{-1}$, since $A_{d} \in H$ and $A_{d} \in S L_{n}(D) \subset N$ (cf. [3]), we conclude that $A_{d} \in P M P^{-1}$ for any $d \in D^{*}$,and so the result follows. Otherwise, we have $H \cap N=N$. Since $S L_{n}(D) \subseteq N$ we conclude that $S L_{n}(D) \subseteq H$ which is clearly a contradiction.

We observe that the above lemma holds also for a maximal subgroup of a subnormal subgroup in $G L_{n}(D)$, as it is known that for $n \geq 2$ subnormal subgroups of $G L_{n}(D)$ are normal, and for $n=1$ the conclusion is clear.

Lemma 2. If $G$ is an irreducible subgroup of $G L_{n}(D)$, then $C_{M_{n}(D)}(G)$ is a division ring.

Proof. It is clear that $C_{M_{n}(D)}(G)$ is a ring. Given $0 \neq X \in C_{M_{n}(D)}(G)$, we may view $X$ as a transformation of the row vectors $D^{n}$. By definition, $\operatorname{Ker} X$ is a $D-G$ bisubmodule of $D^{n}$. Since $G$ is irreducible we conclude that $\operatorname{Ker} X=0$ or $D^{n}$. Thus, we have $\operatorname{Ker} X=0$ because $X \neq 0$. By a similar argument and using the fact the $X$ commutes with each elements of $M_{n}(D)$ one may easily show that $\operatorname{Img} X=D^{n}$, and hence $C_{M_{n}(D)}(G)$ is a division ring.

Lemma 3. If $n \geq 2$ and $M$ satisfies a group identity, then $C_{M_{n}(D)}(M)$ is a field.

Proof. By Lemma 1, we know that $M$ is either irreducible or there exist $P \in$ $G L_{n}(D)$ such that $P^{-1} A_{d} P \in M$ for any $d \in D^{*}$. If the second case occurs, then one may easily show that $D^{*}$ satisfies a group identity and hence, by a theorem of $[15$, p.304], we conclude that $D$ is a field which is a contradiction. Therefore, we may assume that $M$ is irreducible. Now, by Lemma 2, we conclude that $C_{M_{n}(D)}(M)=$ $D_{1}$ is a division ring. Let $x$ be in the derived group of $D_{1}$, i.e., $x \in D_{1}^{\prime}$. Since $S L_{n}(D) \subseteq N$ we conclude that $x \in N$. Now, by maximality of $M$, we have either $\langle x, M\rangle=M$ or $\langle x, M\rangle=N$. In the first case we have $x \in M \cap D_{1}$ and so $x \in Z(M)$. In the second case we obtain $x \in Z(N)$. Now, it is known that $N$ as a non-central 
normal subgroup of $G L_{n}(D)$ with $n \geq 2$ must contain $S L_{n}(D)$ [3]. Therefore, $Z(N) \subseteq F^{*}$, i.e., $x \in Z(N) \subseteq F^{*}$. Hence in any case we have $x \in F^{*} Z(M)$, and so $D_{1}^{\prime} \subseteq F^{*} Z(M)$. This means that $D_{1}^{\prime}$ is abelian and hence, by a result of Hua [10, p. 223], we conclude that $D_{1}$ is a field.

One of the consequences of the above lemma is the following result which will be used later on.

Lemma 4. If $n \geq 2$ and $M$ soluble, then $C_{M_{n}(D)}(M)$ is a field.

The next result essentially says that if $D$ is infinite, then there exists no nonabelian maximal subgroup $M$ of $N$ such that $\left|M / M \cap F^{*}\right|<\infty$.

Theorem 1. Assume the notations of section 2. Then there exists no non-abelian maximal subgroup $M$ of $N$ such that $\left|M / M \cap F^{*}\right|<\infty$.

Proof. Assume first that $F^{*} \subseteq M$, where $M$ is a nonabelian maximal subgroup of $N$. If $n=1$, set $D_{1}=F[M]$. Since $\left|M / F^{*}\right|<\infty$ it is clear that $D_{1}$ is a finite dimensional division algebra. If $N \subseteq D_{1}$, by Cartan-Brauer-Hua Theorem, we obtain $D_{1}=D$. Therefore, $D$ is a finite dimensional division algebra. Let $x_{1}, \cdots, x_{t}$ be the representatives for cosets of $F^{*}$ in $M$, i.e., $M=F^{*} x_{1} \cup \cdots \cup F^{*} x_{t}$. Then, we have $M=\left\langle x_{1}, \cdots, x_{t}\right\rangle F^{*}$, where $\left\langle x_{1}, \cdots, x_{t}\right\rangle$ is the group generated by $x_{1}, \cdots, x_{t}$. Take $x \in N \backslash M$. By maximality of $M$, we obtain $N=\left\langle x_{1}, \cdots, x_{t}, x\right\rangle F^{*}$. Put $H=\left\langle x_{1}, \cdots, x_{t}, x\right\rangle$ so that $N=H F^{*}$. Therefore, $N^{\prime}=H$ is a normal subgroup in $D^{*}$. Now, by Corollary 1 of [13], we conclude that $N^{\prime} \subseteq F^{*}$. This implies that $N$ is central which is a contradiction. Therefore, we must have $N \nsubseteq D_{1}$. Now, by maximality of $M$ we obtain $N \cap D_{1}=M$. It is clear that $M=D_{1} \cap N$ is a subnormal subgroup of $D_{1}^{*}$. Since $\left|M / F^{*}\right|<\infty$, by a result of Herstien [8], we obtain $M \subseteq Z\left(D_{1}\right)$ which is a contradiction to our assumption.

We now assume that $n \geq 2$. By Lemma 1 , we conclude that either $M$ is irreducible or there exists $P \in G L_{n}(D)$ such that $P^{-1} A_{d} P \in M$ for all $d \in D^{*}$. If the second case occurs, we obtain $A_{d}^{t}=a I$, where $a \in F^{*}$ and $t=\left[M: F^{*}\right]$. This in turn implies that $d^{2 t}=1$. Therefore, by a result of [10, p. 225], we conclude 
that $D$ is finite which is a contradiction. So we may assume the first case, i.e., $M$ is irreducible. By a theorem of $[18$, p. 14], we obtain that $F[M]$ is a prime ring with $[F[M]: F]<\infty$. If $F^{*}$ is finite, then so is $M$. Now, by Theorem 4 of [1], which assert that a normal subgroup of $G L_{n}(D)$ does not contain any finite maximal subgroup, we arrive at a contradiction. So, assume that $F^{*}$ is infinite and set $K=C_{M_{n}(D)}(M)$. By Lemma 3 , we know that $K$ is a field. Therefore, $Z(M)$ is an integral domain. Since $[F[M]: F]<\infty$ we conclude that $Z(M)$ is field. Therefore, by a result of [15], $F[M]$ is simple ring. Thus, by Centralizer Theorem, we have $M_{n}(D) \otimes_{F} F[M]^{o p} \simeq K \otimes_{F} M_{s}(F) \simeq M_{s}(K)$, for some positive integer $s$. Therefore, $M_{n}(D)$ is a PI-ring and hence, by a result of Kaplansky, $D$ is a finite dimensional division algebra over its center. Now, let $x_{1}, \cdots, x_{t}$ be a set of representatives for cosets of $F^{*}$ in $M$, i.e, $M=F^{*} x_{1} \cup \cdots \cup F^{*} x_{t}$. Then, we have $M=\left\langle x_{1}, \cdots, x_{t}\right\rangle F^{*}$, where $\left\langle x_{1}, \cdots, x_{t}\right\rangle$ is the group generated by $x_{1}, \cdots, x_{t}$. Take $x \in N \backslash M$. By maximality of $M$, we obtain $N=\left\langle x_{1}, \cdots, x_{t}, x\right\rangle F^{*}$. Put $H=\left\langle x_{1}, \cdots, x_{t}, x\right\rangle$ so that $N=H F^{*}$. This implies that $N^{\prime}=H$ is a normal subgroup of $G L_{n}(D)$. Now, by Corollary 1 of [13], we conclude that $N^{\prime} \subseteq F^{*}$ and hence $N$ is central, which is a contradiction and so the result follows in this case.

Finally, if $F^{*}$ is not a subset of $M$, set $M_{1}=M F^{*}$ and $N_{1}=N F^{*}$. It is clearly seen that $M_{1}$ is a maximal subgroup of $N_{1}$. If $\left|M / M \cap F^{*}\right|$ is finite, then so is $\left|M_{1} / F^{*}\right|$. Therefore, this reduces to the first case and so the result follows.

As a consequence of the above theorem, setting $N=G L_{n}(D)$, we obtain a short proof of Lemma 1 in [12], i.e.,

Corollary 1. Let $D$ be a division ring not necessarily of finite dimension over its center $F$. If either $n=1$ and $D$ is noncommutative or $n>1$ and $D$ infinite, then there exists no maximal subgroup $M$ of $G L_{n}(D), n \geq 1$, containing $F^{*}$ such that $\left|M / F^{*}\right|<\infty$.

Proof. By Theorem 1, there exists no nonabelian maximal subgroup with the stated property. If $M$ is abelian, by Lemma 3, we conclude that $K=C_{M_{n}(D)}(M)$ is a field. Now, by maximality of $M$, we obtain $M=K^{*}$, and hence $K^{*} / F^{*}$ is finite. But it is known that this is not possible unless $M=K^{*}$ is finite. Now, by Corollary 3.11 of [9], we conclude that $D=F$ and $D$ is finite, which is a contradiction. 
The next result provides us with a criterion for $M$ to be nilpotent.

Theorem 2. Let $D$ be a noncommutative finite dimensional F-central division algebra. Then, $M$ is nilpotent if and only if $M$ is contained in the multiplicative group of a subfield of $M_{n}(D)$.

Proof. One way is clear. Assume that $M$ is nilpotent and $n \geq 2$. By Lemma 1 , either $M$ is irreducible or there exists $P \in G L_{n}(D)$ such that $P^{-1} A_{d} P \in M$ for any $d \in D^{*}$. If the second case happens, then one may easily conclude that $D^{*}$ is soluble. Therefore, by Hua's Theorem [10, p. 223], $D$ is commutative which is a contradiction. Therefore, we may assume that $M$ is irreducible. By a result of [18, p. 14], $F[M]$ is a prime ring. Set $K=C_{M_{n}(D)}(M)$. By Lemma 4, we know that $K$ is field. Therefore, $Z(M)$ is an integral domain. Since $[F[M]: F]<\infty$ we conclude that $Z(M)$ is field. Now, by a result of [15, p. 47$], F[M]$ is a simple ring. Thus, by Artin-Wedderburn's Theorem, we obtain $F[M] \simeq M_{n_{1}}\left(D_{1}\right)$ for some positive integer $n_{1}$ and a division ring $D_{1}$. Since $S L_{n}(D) \subseteq N$, we have $S L_{n_{1}}\left(D_{1}\right) \subseteq$ $\left(G L_{n}(D)^{\prime}=S L_{n}(D) \subseteq N\right.$. If $S L_{n_{1}}\left(D_{1}\right) \subseteq M$, then, by a theorem of [18, p.154], we conclude that $D_{1}$ is a locally finite field. This implies that $F$ is a locally finite. Therefore, $D$ is algebraic over a finite field and hence, by Jacobson's Theorem $[10$, p.219], $D$ is commutative which is a contradiction. Thus, $S L_{n_{1}}\left(D_{1}\right) \nsubseteq M$ and by maximality of $M$ in $N$ we must have $M S L_{n_{1}}\left(D_{1}\right)=N$. Since $S L_{n_{1}}\left(D_{1}\right) \subseteq F[M]$ we have $N \subseteq F[M]$ and hence by Cartan-Brauer-Hua Theorem [14], we obtain $F[M]=M_{n}(D)$. Therefore, one may easily show that $Z(M)=M \cap F^{*}$. Since $D$ is of finite dimension over $F$, we may view $M$ as an irreducible nilpotent linear group. Therefore, by a theorem of $[19$, p. 57], we obtain $[M: Z(M)]<\infty$. Now, by Theorem 1, we conclude that $M$ is abelian and so $M \subseteq K=C_{M_{n}(D)(M)}$ and the result follows in this case. It remains to show that the result also holds for $n=1$. So, let $n=1$ and set $D_{1}=F[M]$. If $N \subseteq D_{1}^{*}$, then, by Cartan-Brauer-Hua Theorem, we have $F[M]=D$. Therefore, we obtain $Z(M)=M \cap F^{*}$. Now, by the same argument as used above, we conclude that $M$ is abelian and so the result follows in this case. Finally, assume that $N \nsubseteq D_{1}$. Thus, by maximality of $M$, we have $N \cap D_{1}=M$. It is clear that $D_{1} \cap N \triangleleft D_{1}^{*}$ and so $M \triangleleft D_{1}^{*}$. Now, we know that any noncentral normal subgroup of a finite dimensional division algebra contains a noncyclic free subgroup [7]. Therefore, $M$ as a noncentral normal subgroup of $D_{1}^{*}$ 
contains a noncyclic free subgroup. This is in contradiction with the fact that $M$ is nilpotent and hence result follows.

Now, as a consequence of the above result, we may easily obtain the following corollary which is the last part of Proposition 2 in [12].

Corollary 2. Let $D$ be a noncommutative finite dimensional F-central division algebra, and let $M$ be a maximal subgroup of $G L_{n}(D)$ with $n \geq 1$. Then, $M$ is nilpotent if and only if $M$ is the multiplicative group of a maximal subfield of $M_{n}(D)$.

Proof. Set $N=G L_{n}(D)$. By Theorem 2, $M \subseteq K^{*} \subseteq G L_{n}(D)$, where $K$ is a subfield of $M_{n}(D)$. Now, by maximality of $M$, the result follows.

Given a maximal subgroup $M$ of $G L_{n}(D)$, it is proved in [12] that either $M$ contains a noncyclic free subgroup or there exists a finite family $\left\{K_{i}\right\}_{1}^{r}$ of fields with $F^{*} \subset K_{i}^{*} \subset M$ for all $1 \leq i \leq r$ such that $M / A$ is finite if $C h a r F=0$ and $M / A$ is locally finite if $\operatorname{Char} F=p>0$, where $A=K_{1}^{*} \times \cdots \times K_{r}^{*}$. Now, let $N$ be a normal subgroup of $G L_{n}(D)$ with $n \geq 1$, and $M$ be a maximal subgroup of $N$. In the next result we essentially generalize the above mentioned result to maximal subgroups of $N$ as follows:

Theorem 3. Let $D$ be a noncommutative finite dimensional F-central division algebra, and $N$ be a non-central normal subgroup of $G L_{n}(D)$ with $n \geq 1$. Given a maximal subgroup $M$ of $N$, then, either $M$ contains a noncyclic free subgroup or there exist an abelian subgroup $A$ and a finite family $\left\{K_{i}\right\}_{1}^{r}$ of fields properly containing $F$ with $K_{i}^{*} \subset M$ for all $1 \leq i \leq r$ such that $M / A$ is finite if Char $F=0$ and $M / A$ is locally finite if Charf $=p>0$, where $A \subseteq K_{1}^{*} \times \cdots \times K_{r}^{*}$.

Proof. We first consider the case $n=1$. If $M$ is abelian, then as in the proof of last corollary, $M$ is the multiplicative group of a subfield of $D$ and so the result follows. So, we may assume that $M$ is nonabelian. Set $E=F[M]$. By maximality of $M$, we have either $N \cap E^{*}=M$ or $N \subseteq E^{*}$. If the first case occurs, we conclude that $M$ is normal in $E^{*}$. Therefore, by a result of [7], $M$ contains a noncyclic free 
subgroup. If the second case happens, by Cartan-Brauer-Hua Theorem, we have $F[M]=D$. Assume that $M$ does not contain a noncyclic free subgroup and consider the following cases.

Case 1: $C h a r F=0$. Since $M$ does not contain a noncyclic free subgroup we conclude, by Theorem 1 of [22], that $M$ contains a soluble normal subgroup $T$ of finite index, i.e., $[M: T]<\infty$. If $T \subset F^{*}$, then we obtain $\left[M: F^{*}\right]<\infty$ which contradicts Theorem 1 . Now, $T$ as a subgroup of $D^{*}$ is a completely reducible linear group. Therefore, by a theorem of [19, p. 154], $T$ is abelian-by-finite. Thus, $M$ contains an abelian normal subgroup $A$, say, of finite index. If $A \subset F^{*}$, then we obtain $\left[M: F^{*}\right]<\infty$ which contradicts Theorem 1. Therefore, $A$ is noncentral and $A \subseteq F[A]^{*}=K_{1}^{*}$, where $K_{1}$ is a field and so the result follows in this case.

Case 2: $C h a r F=p>0$. Since $M$ does not contain a noncyclic free subgroup, by Tit's Theorem [22], we conclude that every finitely generated subgroup of $M$ contains a soluble normal subgroup of finite index. Therefore, by a result of Wehrfritz [23], $M / \operatorname{Solv}(M)$ is a torsion linear group, where $\operatorname{Solv}(M)$ is the unique maximal soluble normal subgroup obtained by Zassenhaus-Maltsev Theorem [24]. Therefore, by Schur's Theorem, $M / \operatorname{Solv}(M)$ is locally finite. Set $S=\operatorname{Solv}(M)$. Now, as in the above case, $S$ contains an abelian normal subgroup $B$ of finite index. Therefore, $M / B$ is locally finite and we have $B \subseteq F[B]=K$ which completes the proof of this case.

Now, assume that $n>1$. If $M$ is abelian, by Theorem 2 , the result follows. So, we may assume that $M$ is nonabelian. By Lemma 1, we have that either $M$ is irreducible or there exists $P \in G L_{n}(D)$ such that $P^{-1} A_{d} P \in M$ for any $d \in D^{*}$. If the second case occurs, then $M$ contains a copy of $D^{*}$. Now, by a result of [6], we know that $D^{*}$ contains a noncyclic free subgroup and hence so does $M$. If the first case happens, by Theorem [18, p. 9], we conclude that $F[M]$ is prime ring. Now, by Lemma $2, C=C_{M_{n}(D)}(M)$ is a division ring. We note that $Z(F[M]) \subseteq C$. Therefore, $Z(F[M])$ is an integral domain. Since $[Z(F[M]): F]<\infty$ we conclude that $Z(F[M])$ is field. Now, $F[M]$ is a prime PI-ring and $Z(F[M])$ is a field. Thus, 
by a result of $[15$, p. 47], $F[M]$ is simple ring and hence by Artin-Wedderburn's Theorem, we have $F[M] \simeq M_{n_{1}}\left(D_{1}\right)$, for some positive integer $n_{1}$ and a division ring $D_{1}$. Since $S L_{n}(D) \subseteq N$ we have $S L_{n_{1}}\left(D_{1}\right) \subseteq\left(G L_{n}(D)^{\prime}=S L_{n}(D) \subseteq N\right.$. By maximality of $M$ in $N$, we may consider two cases.

Case 1: $S L_{n_{1}}\left(D_{1}\right) \subseteq M$. If $n_{1}>1$, by Theorem [18, p.154], either $S L_{n_{1}}\left(D_{1}\right)$ contains a noncyclic free subgroup, or $D_{1}$ is a locally finite field. In the first case $M$ contains a noncyclic free subgroup and so the result follows. The second case implies that $F$ is locally finite. This in turn asserts that $D$ is algebraic over a finite field and hence, by Jacobson's Theorem [10, p.219], we conclude that $D$ is a field which is a contradiction. If $n_{1}=1$, then $D_{1}$ is noncommutative since otherwise $M$ is abelian which contradicts our assumption. Now, $D_{1}^{\prime}$ as a normal subgroup of $D_{1}^{*}$ contain a noncyclic free subgroup. Therefore, $M$ contain noncyclic free subgroup and so the result follows in this case.

Case 2: $S L_{n_{1}}\left(D_{1}\right) \nsubseteq M$. By maximality of $M$, we have $M S L_{n_{1}}\left(D_{1}\right)=N$. Since $M$ and $S L_{n_{1}}\left(D_{1}\right)$ are contained in $F[M]$ we have $N \subseteq F[M]$. Now, by CartanBrauer-Hua Theorem [14], we have $F[M]=M_{n}(D)$. If $M$ does not contain a noncyclic free subgroup, we may consider the following subcases.

Subcase 1: $C h a r F=0$. Since $M$ does not contain a noncyclic free subgroup we conclude, by Theorem 1 of [22], that $M$ contains a soluble normal subgroup $T$ of finite index, i.e. $[M: T]<\infty$. By Theorem [18, p. 14], $F[T]$ is semisimple. Since $T$ is a completely reducible linear group, by a result of [19, p. 154], $T$ is abelian-by-finite. Therefore, $M$ contains an abelian normal subgroup $A$, say, of finite index. Now, by a theorem of $[18$, p. 14], $F[A]$ is commutative semisimple ring. Therefore, by Artinwedderburn's Theorem, there exists a finite family $\left\{K_{i}\right\}_{1}^{r}$ of fields with $F^{*} \subset K_{i}^{*}$ for all $1 \leq i \leq r$ such that $F[A]^{\simeq} K_{1} \times \cdots \times K_{r}$. Thus, $A \subseteq F[A]^{*} \simeq K_{1}^{*} \times \cdots \times K_{r}^{*}$, and so the result follows in this case.

Subcase 2: CharF $=p>0$. Since $M$ does not contain a noncyclic free subgroup, by Tit's Theorem [22], we conclude that every finitely generated subgroup 
of $M$ contains a soluble normal subgroup of finite index. Therefore, by a result of Wehrfritz [23], $M / \operatorname{Solv}(M)$ is a torsion linear group, where $\operatorname{Solv}(M)$ is the unique maximal soluble normal subgroup obtained by Zassenhaus-Maltsev Theorem [24]. Therefore, by Schur's Theorem, $M / \operatorname{Solv}(M)$ is locally finite. Set $S=\operatorname{Solv}(M)$. Now, by a result of $[18$, p. 14], $F[S]$ is semisimple. As in Subcase $1, S$ contains an abelian normal subgroup $B$,say, of finite index. Therefore, $M / B$ is locally finite. By Theorem $[18$, p. 14] $F[B]$ is a commutative semisimple ring. Therefore, as in Subcase $1, B \subseteq F[B]^{*} \simeq K_{1}^{*} \times \cdots \times K_{r}^{*}$, and so the proof is complete.

As a consequence of the above theorem, we may prove the following corollary which is the main result of [12].

Corollary 3. Let $D$ be a noncommutative finite dimensional F-central division algebra. Assume that $M$ is a maximal subgroup of $G L_{n}(D)$ with $n \geq 1$. Then, either $M$ contains a noncyclic free subgroup or there exists a finite family $\left\{K_{i}\right\}_{1}^{r}$ of fields with $F^{*} \subset K_{i}^{*} \subset M$ for all $1 \leq i \leq r$ such that $M / A$ is finite if Char $F=0$ and $M / A$ is locally finite if Charf $=p>0$, where $A=K_{1}^{*} \times \cdots \times K_{r}^{*}$.

Proof. If $M$ does not contain a noncyclic free subgroup, by Theorem 3 and a result of $\left[18\right.$, p. 14], we have $F[A]^{*} \simeq K_{1}^{*} \times \cdots \times K_{r}^{*}$. If $F[A]^{*} \subseteq M$, then the result follows. If $L=F[A]^{*} \nsubseteq M$, by maximality of $M$, we obtain $L M=G L_{n}(D)$. Therefore, $L$ is normal in $G L_{n}(D)$, i.e., $S L_{n}(D) \subset L$. But this contradicts the fact that $S L_{n}(D)$ contains a noncyclic free subgroup and so the result follows.

The first author is indebted to the Institute for Studies in Theoretical Physics and Mathematics (IPM) for partial support (Grant No. 83160044). The second author thanks Professor Ulf Rehmann for his hospitality during his stay at the Bielefeld University in March 2006.

\section{References}

[1] S. Akbari, R. Ebrahimian, H. Momenaee Kermani and A. Salehi Golsefidy, The group of units of an Artinian ring, Algebra Colloquium, 9:1 (2002) 81-88. 
[2] S. Akbari, M. Mahdavi-Hezavehi and M. G. Mahmudi, Maximal subgroups of $G L_{1}(D)$, Journal of Algebra, 217 (1999) 422-433.

[3] E. Artin, Geometric algebra, Interscience Pub., New York, 1957.

[4] K. Chiba, Generalized rational identities of subnormal subgroups of skew fields, Proc. Amer. Math. Soc., 124 (6) (1996) 1649-1653.

[5] P. K. Draxl, Skew fields, LMS Lecture Note Series, No. 81, Cambridge University Press, (1982).

[6] J. Goncalves, and A. Mandel, Are there free groups in division rings, Israel J. Math. Vol. 51, No. 1, (1986), 69-80.

[7] J. Goncalves, Free groups in subnormal subgroups and the residual nilpotence of the group of units of group rings, Can. Math. Bull. 27(1982), 365-370.

[8] I. N. Herstein, Multiplicative commutators in division rings II, Rend. Circ. Mat. Palermo II, 29(1980), 485-489.

[9] D. Kiani and M. Mahdavi-Hezavehi, Identities and maximal subgroups of $G L_{n}(D)$,Algebra Colloquium, Volume 12 (2005), Number 3, 461-470.

[10] T. Y. Lam, A first course in non-commutative rings, GTM, No. 131, SpringerVerlag, (1991).

[11] A. E. Lichtman, On subgroups of the multiplicative group of skew fields, Proc. Amer. Math. Soc. 63(1977), 15-16.

[12] M. Mahdavi-Hezavehi, Tits alternative for maximal subgroups of $G L_{n}(D)$, J. Algeb., 271(2004), 518-528.

[13] M. Mahdavi-Hezavehi, M.G. Mahmudi, S. Yasami, Fintely generated subnormal subgroups of $G L_{n}(D)$ are central, J. Algeb., 225, 517-521 (2000).

[14] Alex Rosenberg, The Cartan-Brauer-Hua Theorem for matrix and local matrix rings, Proc. Amer. Mat. Soc., 7(1956), 891-898.

[15] L. H. Rowen, Polynomial identities in ring theory, 1980. 
[16] L. Rowen, Ring theory, Volume II, Academic Press, INC, (1988).

[17] W. R. Scott, Group theory, Dover Publication, INC, 1964.

[18] M. Shirvani and B. A. F. Wehrfritz, Skew linear group, LMS Lecture Note Series, No. 118, (1986)

[19] D. A. Suprunenko, Soluble and nilpotent linear groups, Translations of Mathematical Monographs, Vol. 9, (1963).

[20] D. A. Suprunenko, Matrix groups, Translations of Math. Mono., Amer. Math. Soc., Providence, Rhode Island, (1976).

[21] D. A. Suprunenko, Matrix groups, Translations of Math. Mono., Amer. Math. Soc., Providence, Rhode Island, (1976).

[22] J. Tits, Free subgroup in linear groups, J. of Algebra, 20, 250-270 (1972).

[23] B. A. F. Wehrfritz, 2-generator conditions in linear groups, Archiv. Math. 22, 237-240 (1971).

[24] A. E. Zalesskii, Linear group, in "Algebra IV", part II( A. I. Kostrikin I. R. Shafarevich, Eds.), Encyclopedia of Mathematics and Science. Springer Verlag, Berlin/New York, 1993.

D. Kiani, Department of Pure Mathematics, Faculty of Mathematics and Computer Science, Amirkabir University of Technology (Tehran Polytechnic), 424, Hafez Ave., Tehran 15914, Tehran, Iran, and Institute for Studies in Theoretical Physics and Mathematics (IPM)

M. Mahdavi-Hezavehi, Department of Mathematical Sciences, Sharif University of Technology, P. O. Box 11365-9415, Tehran, Iran, and Fakultät für Mathematik, Universität Bielefeld, Postfach 100131, D-33501 Bielefeld, Germany 